\title{
Oxygen-vacancy effect on structural, magnetic, and ferroelectric properties in multiferroic $\mathrm{YMnO}_{3}$ single crystals
}

D. P. Chen, Y. Du, X. L. Wang, Z. X. Cheng, S. X. Dou, Z. W. Lin, J. G. Zhu, and B. Xu

Citation: Journal of Applied Physics 111, $07 D 913$ (2012);

View online: https://doi.org/10.1063/1.3676000

View Table of Contents: http://aip.scitation.org/toc/jap/111/7

Published by the American Institute of Physics

\section{Articles you may be interested in}

Correlation between oxygen vacancies and sites of $\mathrm{Mn}$ ions in $\mathrm{YMnO}_{3}$

Applied Physics Letters 106, 062905 (2015); 10.1063/1.4909547

Domain wall conductivity in oxygen deficient multiferroic $\mathrm{YMnO}_{3}$ single crystals

Applied Physics Letters 99, 252107 (2011); 10.1063/1.3671393

A medium frequency transformer with multiple secondary windings for medium voltage converter based wind turbine power generating systems

Journal of Applied Physics 113, 17A324 (2013); 10.1063/1.4795850

Oxygen-vacancy ordering as a fatigue mechanism in perovskite ferroelectrics

Applied Physics Letters 76, 3801 (2000); 10.1063/1.126786

Epitaxially grown $\mathrm{YMnO}_{3}$ film: New candidate for nonvolatile memory devices

Applied Physics Letters 69, 1011 (1998); 10.1063/1.117969

Ferroelectric characterization of highly (0001)-oriented $\mathrm{YMnO}_{3}$ thin films grown by chemical solution deposition Applied Physics Letters 73, 903 (1998); 10.1063/1.122443

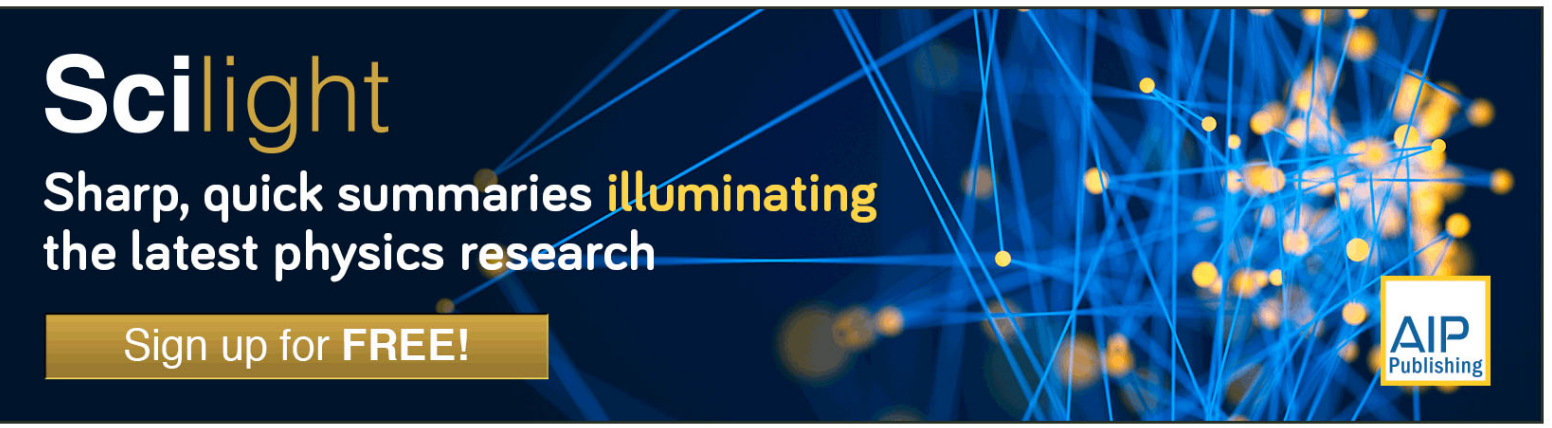




\title{
Oxygen-vacancy effect on structural, magnetic, and ferroelectric properties in multiferroic $\mathrm{YMnO}_{3}$ single crystals
}

\author{
D. P. Chen, ${ }^{1}$ Y. Du, ${ }^{1}$ X. L. Wang, ${ }^{1, \text { a) }}$ Z. X. Cheng,,${ }^{1}$ S. X. Dou, ${ }^{1}$ Z. W. Lin, ${ }^{2}$ J. G. Zhu, ${ }^{2}$ \\ and $\mathrm{B} . \mathrm{Xu}^{3}$ \\ ${ }^{1}$ Institute for Superconducting and Electronic Materials (ISEM), University of Wollongong, Wollongong, \\ New South Wales 2522, Australia \\ ${ }^{2}$ Faculty of Engineering and Information Technology, University of Technology Sydney, Ultimo, New South \\ Wales 2007, Australia \\ ${ }^{3}$ School of Mater. Sci. Engi., Shandong Janzhu University, Jinan 250101, Shandong, China
}

(Presented 2 November 2011; received 26 September 2011; accepted 8 November 2011; published online 2 March 2012)

\begin{abstract}
We have investigated the structural, magnetic, and ferroelectric properties of magnetically frustrated multiferroic $\mathrm{YMnO}_{3}$ single crystals. The ferroelectric domain structures of $\mathrm{YMnO}_{3}$ samples were studied by piezoresponse force microscopy. Instead of domain vortex structure in stoichiometric crystals, $\mathrm{YMnO}_{3-\delta}$ exhibits a random domain configuration with straight domain walls. In magnetic measurements, the $\mathrm{YMnO}_{3-\delta}$ crystal shows typical antiferromagnetic behavior with higher Néel temperature and lower magnetization compared to the stoichiometric sample. The ordered oxygen vacancies dominate multiferroicity through tailoring the domain wall structure. (C) 2012 American Institute of Physics. [doi:10.1063/1.3676000]
\end{abstract}

\section{INTRODUCTION}

Multiferroic materials are attracting much attention due to their coexisting ordered states of electric and magnetic dipoles, which could lead to potential technological applications, such as magnetoelectric random-access memory, by mutual control of magnetism and electricity. ${ }^{1-4}$ Significant progress has been made in the understanding of the origin of multiferroicity in various compounds such as $\mathrm{BiFeO}_{3},{ }^{5-7}$ $\mathrm{Bi}_{2} \mathrm{FeMnO}_{6},{ }^{8}$ and $\mathrm{YMnO}_{3}{ }^{9,10}$ Multiferroic domain walls (DWs), where the ferroic order parameters couple, have been discovered and found to be critical for multiferroicity, which is controllable via material design and external fields. ${ }^{11}$ The defects, including structural, nonstoichiometric, and topological defects, are expected to possess distinct electronic properties at multiferroic DWs, which, in turn, enhances magnetoelectric coupling in multiferroics.

$\mathrm{YMnO}_{3}$, which is crystallized in the hexagonal structure, is a well-known multiferroic compound with large spontaneous polarization, high Curie temperature $(\sim 914 \mathrm{~K})$, and low antiferromagnetic Néel temperature $(\sim 74 \mathrm{~K}){ }^{12-14}$ Very recently, electric dipoles which are induced by $\mathrm{Y} d^{0}$-ness rehybridization together with structural phonon instability were confirmed in $\mathrm{YMnO}_{3}$ by nonlinear optical studies. ${ }^{10}$ Such dipoles indicate the unique structure-transition-driven ferroelectricity in $\mathrm{YMnO}_{3}$. Because structural antiphase boundaries are naturally antiferromagnetic DWs, the ferroelectric DWs tend to pin antiferromagnetic domain boundaries. As a result, structural boundaries, ferroelectric DWs, and antiferromagnetic DWs firmly lock together in hexagonal $\mathrm{YMnO}_{3}$, forming multiferroic DWs. It is expected that the structural defects can lead to new magnetoelectric phe-

\footnotetext{
a) Author to whom correspondence should be addressed. Electronic mail: xiaolin@uow.edu.au.
}

nomena through multiferroic DWs. In this paper, we report ordered oxygen vacancy induced multiferroicity through modifying DWs in oxygen deficient $\mathrm{YMnO}_{3}$ single crystal.

\section{EXPERIMENTAL}

$\mathrm{YMnO}_{3}$ single crystals were grown by the floating zone method (Crystal System Inc.). Two kinds of crystals were grown in air $\left(\mathrm{PO}_{2}=0.1 \mathrm{MPa}\right)$ and $\operatorname{Ar}\left(P_{\mathrm{Ar}}=0.4 \mathrm{MPa}\right)$ atmosphere, which were denoted as the "air-grown" (air-grown $\mathrm{YMnO}_{3}$ ) and "Ar-grown" (Ar-grown $\mathrm{YMnO}_{3-\delta}$ ) samples, respectively.

The crystal structure of samples was examined by powder x-ray diffraction (XRD; GBC MMA). The crystal was ground into powder in order to do XRD refinement. XRD refinement calculation was performed by the Rietica software package (version 1.7.7). Platelet crystals with area of $1 \mathrm{~mm}^{2}$ in the $a b$-plane and thickness of $50 \mu \mathrm{m}$ along the $c$-axis were prepared for piezoresponse force microscopy (PFM, Asylum Research MFP-3 D) observations. Pt/Ir coated Si cantilevers (tip radius $\approx 28 \mathrm{~nm}$ ) with force constant of $2.8 \mathrm{~N} / \mathrm{m}$ were used. The magnetic measurements were carried out using a $14 \mathrm{~T}$ physical properties measurement system (PPMS, Quantum Design).

\section{RESULTS AND DISCUSSION}

Figure 1(a) shows the XRD pattern and refinement calculation results for $\mathrm{YMnO}_{3}$ and $\mathrm{YMnO}_{3-\delta}$ crystals. The diffraction peaks can be indexed well with a hexagonal structure by Joint Committee on Powder Diffraction Standards (JCPDS) Card No. 25-1079. Rietveld refinement results show that $\mathrm{YMnO}_{3-\delta}$ has a unit cell with lattice parameters $a=b=$ $6.137(9) \AA$ and $c=11.411(2) \AA$, which are very close to those of $\mathrm{YMnO}_{3}[a=b=6.138(4) \AA$ and $c=11.407(3) \AA]$. 
The deficiency of oxygen is estimated to be 3\% in Ar-grown $\mathrm{YMnO}_{3-\delta}$ crystal, based on the refinement result.

The oxygen deficiency might possibly lead to Mn ions exhibiting multiple valences in order to maintain the charge balance. As has been reported for other multiferroic compounds, ${ }^{8}$ the valences of transition metals can significantly influence magnetic properties and electric properties. XPS measurements were carried out at room temperature in order to determine the valences of $\mathrm{Mn}$ ions in $\mathrm{YMnO}_{3-\delta}$ crystal, as shown in Fig. 2. The binding energy peaks of Y, Mn, and O have been indexed in the spectra. The XPS spectrum of the Mn $2 p$ region of the sample and the fitting result are shown in the inset. Two main peaks at 653.701 and $642.156 \mathrm{eV}$ correspond to Mn $2 p 1 / 2$ and $2 p 3 / 2$, respectively, which indicates that manganese ions present a valence of +3 in $\mathrm{YMnO}_{3-\delta}$ crystal. The XRD and XPS results confirm that there is no secondary phase formation in the oxygen deficient crystal sample.

Figure 3 shows out-of-plane PFM images of the $a b$ surface of $\mathrm{YMnO}_{3}$ single crystals. A typical wedge-shaped ferroelectric domain structure has been observed in

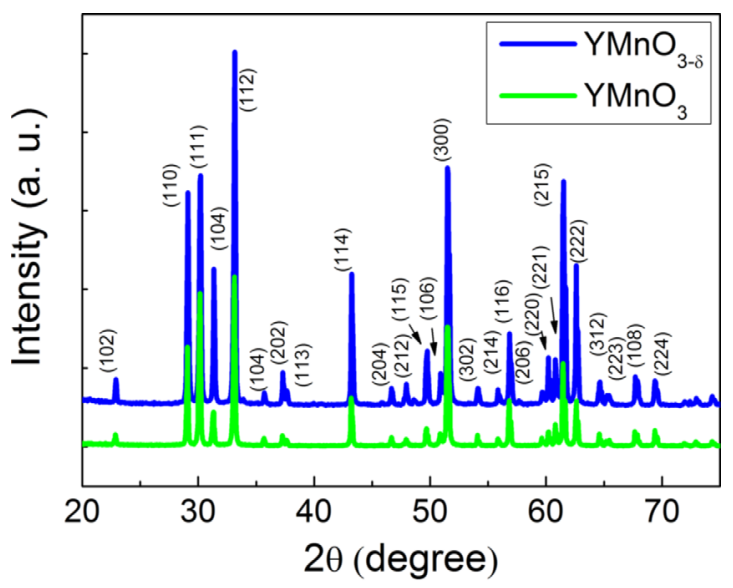

(a)

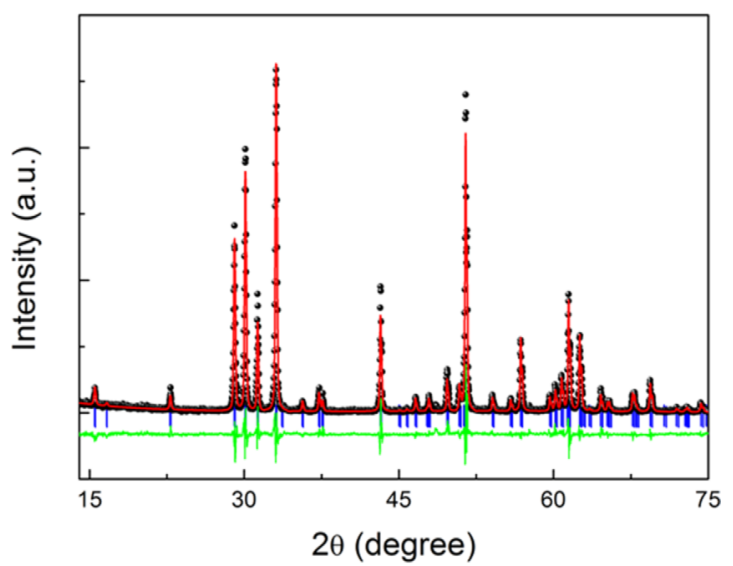

(b)

FIG. 1. (Color online) (a) $\mathrm{XRD}$ patterns for stoichiometric $\mathrm{YMnO}_{3}$ (index) and oxygen deficient $\mathrm{YMnO}_{3-\delta}$ (no index) single crystal samples. (b) XRD refinement calculation results for $\mathrm{YMnO}_{3-\delta}$ crystal samples. The short vertical lines mark the peak positions of the standard, and the curve at the bottom of (b) is the difference spectrum. stoichiometric $\mathrm{YMnO}_{3}$ crystal, as shown in Fig. 3(a). Six different domain phases assigned as $\pm \alpha, \pm \beta$, and $\pm \gamma$ with downward $P_{\downarrow}$ (bright area) and three upward $P_{\uparrow}$ (dark area) polarizations join at a clamping point. The domain size is $0.5-2.0 \mu \mathrm{m}$. Figure 3(b) shows a PFM image of the $a b$ surface of Ar-grown $\mathrm{YMnO}_{3-\delta}$ crystal. Interestingly, the domain structures of the Ar-grown $\mathrm{YMnO}_{3-\delta}$ crystal are notably distinct from that of the air-grown sample. The domain sizes in the Ar-grown $\mathrm{YMnO}_{3-\delta}$ are in the range of $2.0-8.0 \mu \mathrm{m}$, which is four times larger than was observed for the air-grown samples. Instead of domain vortices as in the stoichiometric sample, the domain structure exhibits random shapes in $\mathrm{YMnO}_{3-\delta}$, with one or more straight DW. At high concentration, oxygen vacancies tend to be ordered as pinning centers at DWs, forming vacancy chains and planes in ferroelectrics ${ }^{15,16}$ such as $\mathrm{SrTiO}_{3} \cdot{ }^{17}$ In our sample, the concentration of oxygen vacancy reaches as high as $\sim 3 \%$. It is proposed that the straight DWs are induced by ordered oxygen vacancies in our $\mathrm{YMnO}_{3-\delta}$.

Figure 4 shows the field-cooling magnetization $(M)$ as a function of temperature (T) for $\mathrm{YMnO}_{3}$ and $\mathrm{YMnO}_{3-\delta}$ crystals measured in the temperature range from 5 to $300 \mathrm{~K}$ in a magnetic field of 1000 Oe. Compared to the $\mathrm{YMnO}_{3}$ crystal, the oxygen deficient $\mathrm{YMnO}_{3-\delta}$ sample shows lower magnetization from 5 to $300 \mathrm{~K}$. The magnetic transitions were observed at $T_{1}=73 \mathrm{~K}$ and $T_{2}=78 \mathrm{~K}$ for $\mathrm{YMnO}_{3}$ and $\mathrm{YMnO}_{3-\delta}$, respectively. In stoichiometric $\mathrm{YMnO}_{3}, \mathrm{Mn}-\mathrm{O}-\mathrm{Mn}$ is ordered antiferromagnetically through empty $\mathrm{Mn}^{3+} 3 d$ orbitals and $\mathrm{O}^{2-} 2 p$ orbitals. However, the ordered oxygen vacancies at multiferroic DWs in $\mathrm{YMnO}_{3-\delta}$ lead to a local structural distortion, which will result in weak Mn-O-Mn ordering at DWs. As a result, the ordering temperature of $\mathrm{YMnO}_{3-\delta}$ is slightly higher than for the stoichiometric crystal. In order to further confirm the magnetic properties of $\mathrm{YMnO}_{3-\delta}$ crystal, the magnetization as a function of magnetic field $(H)$ was measured in a magnetic field of $5 \mathrm{~T}$ at 10 and $300 \mathrm{~K}$, as shown in the Fig. 4 inset. The linear $M-H$ loops of $\mathrm{YMnO}_{3-\delta}$ indicate that the sample exhibits antiferromagnetic behavior at both low and room temperature.

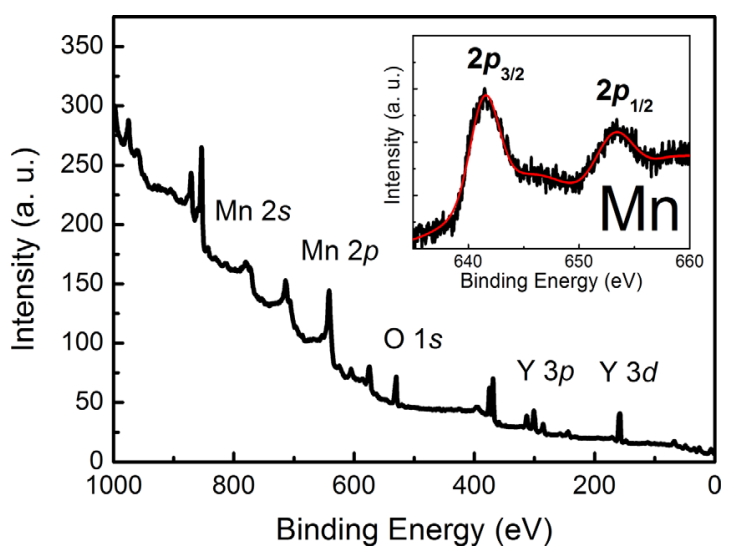

FIG. 2. (Color online) XPS spectra of Ar-grown $\mathrm{YMnO}_{3-\delta}$ crystal. Inset is an enlargement of the Mn $2 p$ region. 
(a)

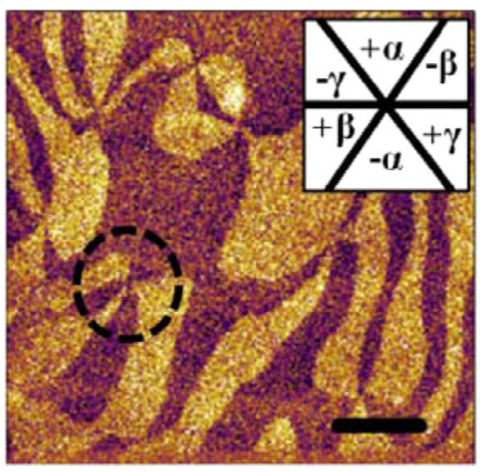

(c)

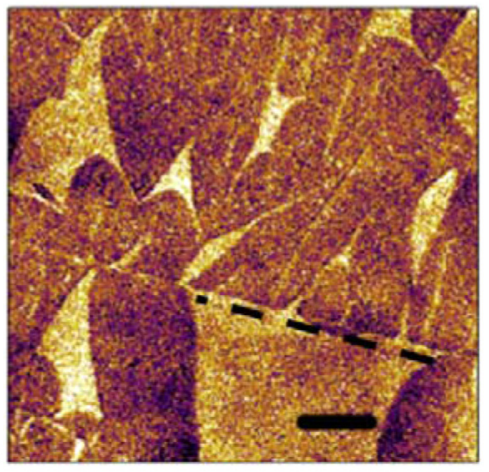

(b)

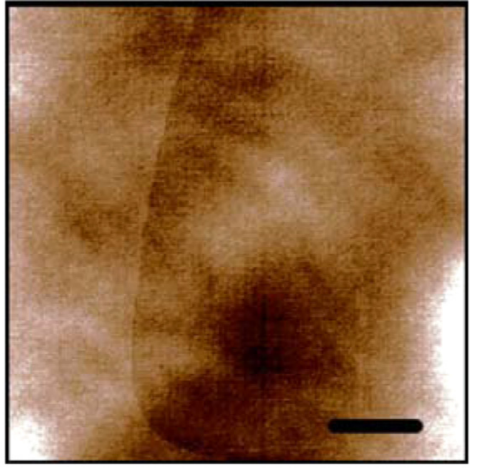

(d)

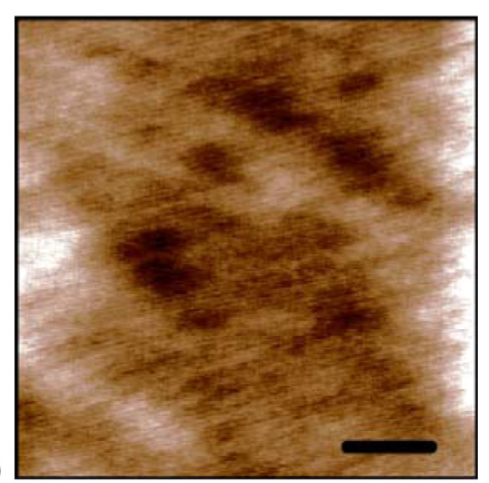

FIG. 3. (Color online) PFM images of the $a-b$ surface of (a) air-grown $\mathrm{YMnO}_{3}$ and (c) Ar-grown $\mathrm{YMnO}_{3-\delta}$ single crystals. The inset in (a) is the domain configuration of six domain phases joined at a clamping point, which is indicated as a dashed circle in (a). The dashed line in (c) shows a straight $\mathrm{DW}$ in $\mathrm{YMnO}_{3-\delta}$ single crystal. Bright and dark areas correspond to the ferroelectric domains with $\mathrm{P}_{\downarrow}$ and $\mathrm{P}_{\uparrow}$, respectively. (b) and (d) are corresponding topography images for (a) and (c). The surface roughness of both crystals is $\sim 2 \mathrm{~nm}$. The scale bars represents $1 \mu \mathrm{m}$ in (a) and (b); and $2 \mu \mathrm{m}$ in (c) and (d).

\section{CONCLUSION}

In summary, the structural, ferroelectric, and magnetic interaction between oxygen vacancies and domain structures has been investigated in oxygen deficient $\mathrm{YMnO}_{3-\delta}$ crystal. The valence of $\mathrm{Mn}$ ions remains +3 , even with the high oxygen vacancy concentration $(\sim 3 \%)$ present in the sample. The ordered oxygen vacancies were verified to induce quasistraight $\mathrm{DWs}$ in $\mathrm{YMnO}_{3-\delta}$ with spontaneous polarization. The Néel temperature and magnetization of the $\mathrm{YMnO}_{3-\delta}$ crystal were found to be lower than those of the stoichiometric $\mathrm{YMnO}_{3}$, which is possibly attributable to the location of structural distortion at DWs induced by oxygen vacancies.

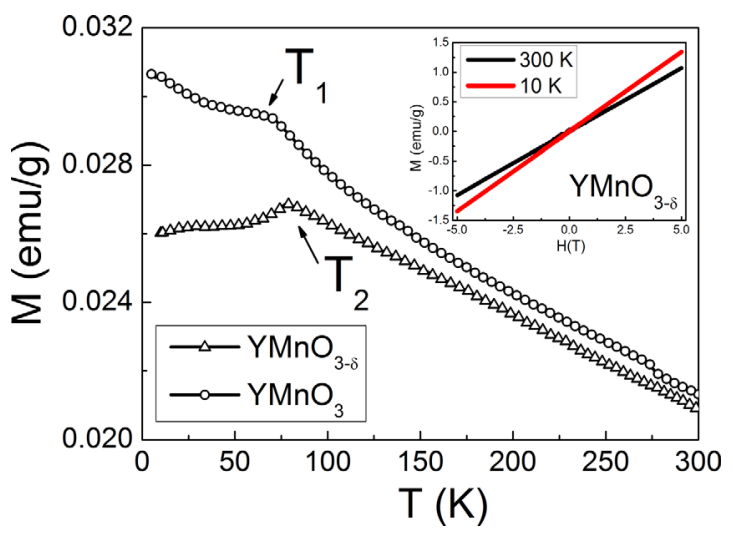

FIG. 4. (Color online) Field cooling magnetization as a function of temperature for air-grown $\mathrm{YMnO}_{3}$ and Ar-grown $\mathrm{YMnO}_{3-\delta}$ single crystals measured in the temperature range from $5 \mathrm{~K}$ to $300 \mathrm{~K}$ in a magnetic field of $1000 \mathrm{Oe}$; the inset contains the $M-H$ loops of Ar-grown $\mathrm{YMnO}_{3-\delta}$ measured at 10 and $300 \mathrm{~K}$.

\section{ACKNOWLEDGMENTS}

This work is supported by the Australian Research Council (ARC) through Discovery projects DP0987190 and DP0558753.

${ }^{1}$ B. K. Ponomarev, S. A. Ivanov, Y. F. Popov, V. D. Negrii, and B. S. Red'kin, Ferroelectrics 161, 43 (1994).

${ }^{2}$ H. Schmid, Ferroelectrics 162, 665 (1994).

${ }^{3}$ N. A. Hill, J. Phys. Chem. B 104, 6694 (2000).

${ }^{4}$ M. Gajek, M. Bibes, A. Barthélémy, K. Bouzehouane, S. Fusil, M. Varela, J. Fontcuberta, and A. Fert, Phys. Rev. B 72, 020406(R) (2005).

${ }^{5}$ J. Wang, J. B. Neaton, H. Zheng, V. Nagarajan, B. Ogale, B. Liu, D. Viehland, V. Vaithyanathan, D. G. Schlom, U. V. Waghmare, N. A. Spaldin, K. M. Rabe, M. Wuttig, and R. Ramesh, Science 299, 1719 (2003).

${ }^{6}$ Y. Du, Z. X. Cheng, S. X. Dou, D. J. Attard, and X. L. Wang, J. Appl. Phys. 109, 073903 (2011).

${ }^{7}$ Y. Chu, Q. He, C. Yang, P. Yu, L. W. Martin, P. Shafer, and R. Ramesh, Nano Lett. 9, 1726 (2009).

${ }^{8}$ Y. Du, Z. X. Cheng, S. X. Dou, and X. L Wang, Appl. Phys. Lett. 97, 122502 (2010).

${ }^{9}$ B. B. V. Aken, T. T. M. Palstra, A. Filippetti, and N. A. Spaldin, Nature Mater. 3, 164 (2004).

${ }^{10}$ T. Choi, Y. Horibe, H. T. Yi, Y. J. Choi, W. Wu, and S. W. Cheong, Nature Mater. 9, 423 (2010).

${ }^{11}$ P. Maksymovych, J. Seidel, Y. H. Chu, P. Wu, A. P. Baddorf, L. Q. Chen, S. V. Kalinin, and R. Ramesh, Nano Lett. 11, 1906 (2011).

${ }^{12}$ D. Y. Cho, J. Y. Kim, B. G. Park, K. J. Rho, J. H. Park, H. J. Noh, B. J. Kim, S. J. Oh, H. M. Park, J. S. Ahn, H. Ishibashi, S. W. Cheong, J. H. Lee, P. Murugavel, T. W. Noh, A. Tanaka, and T. Jo, Phys. Rev. Lett. 98, 217601 (2007).

${ }^{13}$ T. Katsufuji, S. Mori, M. Masaki, Y. Moritomo, N. Yamamoto, and H. Takagi, Phys. Rev. B 64, 104419 (2001).

${ }^{14}$ C. C. Neacsu, B. B. van Aken, M. Fiebig, and M. B. Raschke, Phys. Rev. B 79, 100107 (2009).

${ }^{15}$ J. F. Scott and M. Dawber, Appl. Phys. Lett. 76, 3801 (2000).

${ }^{16}$ Y. Kitanaka, Y. Noguchi, and M. Miyayama, Phys. Rev. B 81, 094114 (2010).

${ }^{17}$ D. D. Cuong, B. Lee, K. M. Choi. H. Ahn, S. Han, and J. Lee, Phys. Rev. Lett. 98, 115503 (2007). 